M. K. Aouf - T. M. Seoudy

\title{
On differential sandwich theorems of $p$-valent analytic functions defined by the integral operator
}

Received: 28 May 2012 / Accepted: 27 November 2012 / Published online: 15 December 2012

(C) The Author(s) 2012. This article is published with open access at Springerlink.com

\begin{abstract}
In this paper, we derive some subordination and superordination results for certain $p$-valent analytic functions in the open unit disc, which are acted upon by an integral operator. Relevant connection of the results, which are presented in this paper with various known results are also considered.
\end{abstract}

\section{Mathematics Subject Classification 30C45}

في هذه الورقة نقوم باثتقاق بعض نتائج تحت التنسيق (subordination) وفوق التنسيق (superordination) للاو ال التحليلية p-متكافئة في قرص وصن الوحدة المفتوح، والتي تمّ التأثير عليها بو اسطة مؤثر تكامل. كما تمّ أيضاً التطرق إلى عدة نتائج معروفة وذات علاقة بنتائجنا المعروضة.

\section{Introduction}

Let $H(U)$ denote the class of analytic functions in the open unit disc $U=\{z \in \mathbb{C}:|z|<1\}$ and let $H[a, p]$ denote the subclass of the functions $f \in H(U)$ of the form:

$$
f(z)=a+a_{p} z^{p}+a_{p+1} z^{p+1}+\cdots \quad(a \in \mathbb{C} ; p \in \mathbb{N}=\{1,2, \ldots\}) .
$$

Also, let $A(p)$ be the subclass of the functions $f \in H(U)$ of the form:

$$
f(z)=z^{p}+\sum_{k=p+1}^{\infty} a_{k} z^{k} \quad(p \in \mathbb{N}),
$$

and set $A \equiv A(1)$. For functions $f(z) \in A(p)$, given by (1.1), and $g(z)$ given by

$$
g(z)=z^{p}+\sum_{k=p+1}^{\infty} b_{k} z^{k} \quad(p \in \mathbb{N}),
$$

M. K. Aouf ( $\varangle)$

Department of Mathematics, Faculty of Science, Mansoura University,

Mansoura 35516, Egypt

E-mail: mkaouf127@yahoo.com

T. M. Seoudy

Department of Mathematics, Faculty of Science, Fayoum University,

Fayoum 63514, Egypt

E-mail: tms00@fayoum.edu.eg 
the Hadamard product (or convolution) of $f(z)$ and $g(z)$ is defined by

$$
(f * g)(z)=z^{p}+\sum_{k=p+1}^{\infty} a_{k} b_{k} z^{k}=(g * f)(z) \quad(z \in U ; p \in \mathbb{N}) .
$$

For $f, g \in H(U)$, we say that the function $f$ is subordinate to $g$, if there exists a Schwartz function $w$, i.e, $w \in H(U)$ with $w(0)=0$ and $|w(z)|<1, z \in U$, such that $f(z)=g(w(z))$ for all $z \in U$. This subordination is usually denoted by $f(z) \prec g(z)$. It is well-known that, if the function $g$ is univalent in $U$, then $f(z) \prec g(z)$ is equivalent to $f(0)=g(0)$ and $f(U) \subset g(U)$ (see [5,6]).

Supposing that $h$ and $k$ are two analytic functions in $U$, let

$$
\varphi(r, s, t ; z): \mathbb{C}^{3} \times U \rightarrow \mathbb{C} .
$$

If $h$ and $\varphi\left(h(z), z h^{\prime}(z), z^{2} h^{\prime \prime}(z) ; z\right)$ are univalent functions in $U$, and if $h$ satisfies the second-order superordination

$$
k(z) \prec \varphi\left(h(z), z h^{\prime}(z), z^{2} h^{\prime \prime}(z) ; z\right),
$$

then $h$ is a solution of the differential superordination (1.4). A function $q \in H(U)$ is called a subordinant of (1.4), if $q(z) \prec h(z)$ for all the functions $h$ satisfying (1.4). A univalent subordinant $\widetilde{q}$ that satisfies $q(z) \prec \widetilde{q}(z)$ for all of the subordinants $q$ of (1.4) is the best subordinant.

Recently, Miller and Mocanu [7] obtained sufficient conditions on the functions $k, q$ and $\varphi$ for which the following implication holds:

$$
k(z) \prec \varphi\left(h(z), z h^{\prime}(z), z^{2} h^{\prime \prime}(z) ; z\right) \Rightarrow q(z) \prec h(z) .
$$

Using these results, Bulboaca [3] considered certain classes of first-order differential superordinations, as well as superordination-preserving integral operators [4]. Ali et al. [1], using the results from [3], obtained sufficient conditions for certain normalized analytic functions to satisfy

$$
q_{1}(z) \prec \frac{z f^{\prime}(z)}{f(z)} \prec q_{2}(z),
$$

where $q_{1}$ and $q_{2}$ are given univalent normalized functions in $U$.

Very recently, Shanmugam et al. [14-17] obtained the sandwich results for certain classes of analytic functions. Further subordination results can be found in $[8,9,13,18-20]$.

For $p \in \mathbb{N}, n \in \mathbb{N}_{0}=\mathbb{N} \cup\{0\}, \lambda>0$ and $f \in A(p)$, we consider the integral operator defined as follows:

$$
\begin{aligned}
& I_{p, \lambda}^{0} f(z)=f(z), \\
& I_{p, \lambda}^{1} f(z)=\frac{p}{\lambda} z^{p-\frac{p}{\lambda}} \int_{0}^{z} t^{\frac{p}{\lambda}-p-1} f(t) \mathrm{d} t=z^{p}+\sum_{k=p+1}^{\infty}\left[\frac{p}{p+\lambda(k-p)}\right] a_{k} z^{k}, \\
& I_{p, \lambda}^{2} f(z)=\frac{p}{\lambda} z^{p-\frac{p}{\lambda}} \int_{0}^{z} t^{\frac{p}{\lambda}-p-1} I_{p, \lambda}^{1} f(t) \mathrm{d} t=z^{p}+\sum_{k=p+1}^{\infty}\left[\frac{p}{p+\lambda(k-p)}\right]^{2} a_{k} z^{k},
\end{aligned}
$$

and (in general)

$$
\begin{aligned}
I_{p, \lambda}^{n} f(z) & =\frac{p}{\lambda} z^{p-\frac{p}{\lambda}} \int_{0}^{z} t^{\frac{p}{\lambda}-p-1} I_{p, \lambda}^{n-1} f(t) \mathrm{d} t=z^{p}+\sum_{k=p+1}^{\infty}\left[\frac{p}{p+\lambda(k-p)}\right]^{n} a_{k} z^{k} \\
& =\underbrace{I_{p, \lambda}^{1}\left(\frac{z^{p}}{1-z}\right) * I_{p, \lambda}^{1}\left(\frac{z^{p}}{1-z}\right) * \cdots * I_{p, \lambda}^{1}\left(\frac{z^{p}}{1-z}\right)}_{n \text {-times }} * f(z),
\end{aligned}
$$

then from (1.5), we can easily deduce that

$$
\frac{\lambda}{p} z\left(I_{p, \lambda}^{n} f(z)\right)^{\prime}=I_{p, \lambda}^{n-1} f(z)-(1-\lambda) I_{p, \lambda}^{n} f(z) \quad(p, n \in \mathbb{N} ; \lambda>0) .
$$

We note that: 
(i) $I_{1, \lambda}^{n} f(z)=I_{\lambda}^{-n} f(z)($ see $[10])$

$$
=\left\{f(z) \in A: I_{\lambda}^{-n} f(z)=z+\sum_{k=2}^{\infty}[1+\lambda(k-1)]^{-n} a_{k} z^{k}\left(n \in \mathbb{N}_{0}\right)\right\} ;
$$

(ii) $I_{1,1}^{n} f(z)=I^{n} f(z)($ see $[12])$

$$
=\left\{f(z) \in A: I^{n} f(z)=z+\sum_{k=2}^{\infty} k^{-n} a_{k} z^{k}\left(n \in \mathbb{N}_{0}\right)\right\} .
$$

Also we note that $I_{p, 1}^{n} f(z)=I_{p}^{n} f(z)$, where $I_{p}^{n}$ is $p$-valent Salagean integral operator

$$
I_{p}^{n} f(z)=\left\{f(z) \in A(p): I_{p}^{n} f(z)=z^{p}+\sum_{k=p+1}^{\infty}\left(\frac{p}{k}\right)^{n} a_{k} z^{k}\left(p \in \mathbb{N}, n \in \mathbb{N}_{0}\right)\right\} .
$$

In this paper, we will derive several subordination results, superordination results and sandwich results involving the operator $I_{p, \lambda}^{n}$.

\section{Preliminaries}

In order to prove our subordination and superordination results, we make use of the following known definition and results.

Definition 2.1 [7] Denote by $Q$ the set of all functions $f(z)$ that are analytic and injective on $\bar{U} \backslash E(f)$, where

$$
E(f)=\left\{\zeta: \zeta \in \partial \text { and } \lim _{z \rightarrow \zeta} f(z)=\infty\right\}
$$

and are such that $f^{\prime}(\zeta) \neq 0$ for $\zeta \in \partial U \backslash E(f)$.

Lemma 2.2 [6] Let the function $q(z)$ be univalent in the unit disc $U$ and let $\theta$ and $\varphi$ be analytic in a domain $D$ containing $q(U)$ with $\varphi(w) \neq 0$ when $w \in q(U)$. Set $Q(z)=z q^{\prime}(z) \varphi(q(z))$ and $h(z)=\theta(q(z))+Q(z)$. Suppose that

(i) $Q(z)$ is starlike univalent in $U$,

(ii) $\Re\left\{\frac{z h^{\prime}(z)}{Q(z)}\right\}>0$ for $z \in U$.

If $p$ is analytic with $p(0)=q(0), p(U) \subseteq D$ and

$$
\theta(p(z))+z p^{\prime}(z) \varphi(p(z)) \prec \theta(q(z))+z q^{\prime}(z) \varphi(q(z)),
$$

then

$$
p(z) \prec q(z)
$$

and $q(z)$ is the best dominant.

Lemma 2.3 [16] Let $q$ be a convex univalent function in $U$ and let $\psi \in \mathbb{C}, \gamma \in \mathbb{C}^{*}=\mathbb{C} \backslash\{0\}$ with

$$
\Re\left\{1+\frac{z q^{\prime \prime}(z)}{q^{\prime}(z)}\right\}>\max \left\{0,-\Re\left(\frac{\psi}{\gamma}\right)\right\} .
$$

If $p(z)$ is analytic in $U$ with $p(0)=q(0)$ and

$$
\psi p(z)+\gamma z p^{\prime}(z) \prec \psi q(z)+\gamma z q^{\prime}(z),
$$

then

$$
p(z) \prec q(z) \quad(z \in U)
$$

and $q$ is the best dominant. 
Lemma 2.4 [5] Let $q(z)$ be convex univalent in the unit disc $U$ and let $\theta$ and $\varphi$ be analytic in a domain $D$ containing $q(U)$. Suppose that

(i) $\Re\left\{\frac{\theta^{\prime}(q(z))}{\varphi(q(z))}\right\}>0$ for $z \in U$;

(ii) $z q^{\prime}(z) \varphi(q(z))$ is starlike univalent in $U$.

If $p(z) \in H[q(0), 1] \cap Q$, with $p(U) \subseteq D$, and $\theta(p(z))+z p^{\prime}(z) \varphi(p(z))$ is univalent in $U$, and

$$
\theta(q(z))+z q^{\prime}(z) \varphi(q(z)) \prec \theta(p(z))+z p^{\prime}(z) \varphi(p(z)),
$$

then

$$
q(z) \prec p(z) \quad(z \in U)
$$

and $q(z)$ is the best subordinant.

Lemma 2.5 [7] Let $q$ be convex univalent in $U$ and $\gamma \in \mathbb{C}$. Further assume that $\Re(\gamma)>0$. If $p(z) \in$ $H[q(0), 1] \cap Q$ and $p(z)+\gamma z p^{\prime}(z)$ is univalent in $U$, then

$$
q(z)+\gamma z q^{\prime}(z) \prec p(z)+\gamma z p^{\prime}(z)
$$

implies

$$
q(z) \prec p(z) \quad(z \in U)
$$

and $q$ is the best subordinant.

The last lemma gives us a necessary and sufficient condition for the univalence of a special function which will be used in some particular case.

Lemma 2.6 [11] The function $q(z)=(1-z)^{-2 a b}$ is univalent in the unit disc $U$ if and only if $|2 a b-1| \leq 1$ or $|2 a b+1| \leq 1$.

\section{Subordination results}

Unless otherwise mentioned, we assume throughout this paper that $\lambda>0, p, n \in \mathbb{N}$ and the powers understood as principal values.

Theorem 3.1 Let $q$ be univalent in $U$, with $q(0)=1$, and suppose that

$$
\Re\left(1+\frac{z q^{\prime \prime}(z)}{q^{\prime}(z)}\right)>\max \left\{0 ;-\frac{p^{2}}{\lambda} \Re\left(\frac{1}{\mu}\right)\right\} \quad\left(\mu \in \mathbb{C}^{*} ; z \in U\right) .
$$

If $f \in A(p)$ satisfies the following subordination condition

$$
\frac{\mu}{p}\left(\frac{I_{p, \lambda}^{n-1} f(z)}{z^{p}}\right)+\frac{p-\mu}{p}\left(\frac{I_{p, \lambda}^{n} f(z)}{z^{p}}\right) \prec q(z)+\frac{\mu \lambda}{p^{2}} z q^{\prime}(z),
$$

then

$$
\frac{I_{p, \lambda}^{n} f(z)}{z^{p}} \prec q(z)
$$

and $q$ is the best dominant of (3.2). 
Proof If we consider the analytic function

$$
h(z)=\frac{I_{p, \lambda}^{n} f(z)}{z^{p}} \quad(z \in U),
$$

by differentiating (3.4) logarithmically with respect to $z$, we deduce that

$$
\frac{z h^{\prime}(z)}{h(z)}=\frac{z\left(I_{p, \lambda}^{n} f(z)\right)^{\prime}}{I_{p, \lambda}^{n} f(z)}-p .
$$

From (3.5), by using the identity (1.6), a simple computation shows that

$$
\frac{\mu}{p}\left(\frac{I_{p, \lambda}^{n-1} f(z)}{z^{p}}\right)+\frac{p-\mu}{p}\left(\frac{I_{p, \lambda}^{n} f(z)}{z^{p}}\right)=h(z)+\frac{\lambda \mu}{p^{2}} z h^{\prime}(z),
$$

hence the subordination (3.2) is equivalent to

$$
h(z)+\frac{\lambda \mu}{p^{2}} z h^{\prime}(z) \prec q(z)+\frac{\lambda \mu}{p^{2}} z q^{\prime}(z) .
$$

An application of Lemma 2.3, with $\psi=1$ and $\gamma=\frac{\lambda \mu}{p^{2}}$, leads to (3.3).

Taking $q(z)=\frac{1+A z}{1+B z}(-1 \leq B<A \leq 1)$ in Theorem 3.1, the condition (3.1) becomes

$$
\Re\left(\frac{1-B z}{1+B z}\right)>\max \left\{0 ;-\frac{p^{2}}{\lambda} \Re\left(\frac{1}{\mu}\right)\right\} \quad(z \in U) .
$$

It is easy to check that the function $\varphi(\zeta)=\frac{1-\zeta}{1+\zeta},|\zeta|<|B|$, is convex in $U$, and since $\varphi(\bar{\zeta})=\overline{\varphi(\zeta)}$ for all $|\zeta|<|B|$, it follows that the image $\phi(U)$ is a convex domain symmetric with respect to the real axis, hence

$$
\inf \left\{\Re\left(\frac{1-B z}{1+B z}\right): z \in U\right\}=\frac{1-|B|}{1+|B|}>0
$$

Then, the inequality (3.7) is equivalent to

$$
\frac{p^{2}}{\lambda} \Re\left(\frac{1}{\mu}\right) \geq \frac{|B|-1}{|B|+1},
$$

hence we obtain the following result.

Corollary 3.2 Let $\mu \in \mathbb{C}^{*}$ and

$$
\max \left\{0 ;-\frac{p^{2}}{\lambda} \Re\left(\frac{1}{\mu}\right)\right\} \leq \frac{1-|B|}{1+|B|} .
$$

If $f \in A(p)$ satisfies the following subordination condition

$$
\frac{\mu}{p}\left(\frac{I_{p, \lambda}^{n-1} f(z)}{z^{p}}\right)+\frac{p-\mu}{p}\left(\frac{I_{p, \lambda}^{n} f(z)}{z^{p}}\right) \prec \frac{1+A z}{1+B z}+\frac{\mu \lambda}{p^{2}} \frac{(A-B) z}{(1+B z)^{2}}
$$

then

$$
\frac{I_{p, \lambda}^{n} f(z)}{z^{p}} \prec \frac{1+A z}{1+B z}
$$

and $\frac{1+A z}{1+B z}$ is the best dominant of (3.9).

For $p=A=-B=1$, the above corollary reduces as follows: 
Example 3.3 Let $\mu \in \mathbb{C}^{*}$ with $\Re\left(\frac{1}{\mu}\right) \geq 0$. If $f \in A$ satisfies the following subordination condition

$$
\mu\left(\frac{I_{\lambda}^{n-1} f(z)}{z}\right)+(1-\mu)\left(\frac{I_{\lambda}^{n} f(z)}{z}\right) \prec \frac{1+z}{1-z}+\frac{2 \mu \lambda z}{(1-z)^{2}},
$$

then

$$
\frac{I_{\lambda}^{n} f(z)}{z} \prec \frac{1+z}{1-z}
$$

and $\frac{1+z}{1-z}$ is the best dominant of (3.10).

Theorem 3.4 Let $q(z)$ be univalent in $U$, with $q(0)=1$ and $q(z) \neq 0$ for all $z \in U$. Let $\gamma, \mu \in \mathbb{C}^{*}$ and $v, \eta \in \mathbb{C}^{*}$ with $v+\eta \neq 0$. Let $f \in A(p)$ and suppose that $f$ and $q$ satisfy the next conditions:

$$
\frac{\nu I_{p, \lambda}^{n-1} f(z)+\eta I_{p, \lambda}^{n} f(z)}{(v+\eta) z^{p}} \neq 0 \quad(z \in U),
$$

and

$$
\Re\left(1+\frac{z q^{\prime \prime}(z)}{q^{\prime}(z)}-\frac{z q^{\prime}(z)}{q(z)}\right)>0 \quad(z \in U)
$$

If

$$
1+\gamma \mu\left[\frac{\nu z\left(I_{p, \lambda}^{n-1} f(z)\right)^{\prime}+\eta z\left(I_{p, \lambda}^{n} f(z)\right)^{\prime}}{\nu I_{p, \lambda}^{n-1} f(z)+\eta I_{p, \lambda}^{n} f(z)}-p\right] \prec 1+\gamma \frac{z q^{\prime}(z)}{q(z)}
$$

then

$$
\left[\frac{v I_{p, \lambda}^{n-1} f(z)+\eta I_{p, \lambda}^{n} f(z)}{(v+\eta) z^{p}}\right]^{\mu} \prec q(z)
$$

and $q$ is the best dominant of (3.13).

Proof Let

$$
h(z)=\left[\frac{\nu I_{p, \lambda}^{n-1} f(z)+\eta I_{p, \lambda}^{n} f(z)}{(\nu+\eta) z^{p}}\right]^{\mu} \quad(z \in U) .
$$

According to (3.11) the function $h(z)$ is analytic in $U$, and differentiating (3.14) logarithmically with respect to $z$, we obtain

$$
\frac{z h^{\prime}(z)}{h(z)}=\mu\left[\frac{\nu z\left(I_{p, \lambda}^{n-1} f(z)\right)^{\prime}+\eta z\left(I_{p, \lambda}^{n} f(z)\right)^{\prime}}{\nu I_{p, \lambda}^{n-1} f(z)+\eta I_{p, \lambda}^{n} f(z)}-p\right] .
$$

In order to prove our result we will use Lemma 2.2. In this lemma consider

$$
\theta(w)=1 \text { and } \varphi(w)=\frac{\gamma}{w},
$$

then $\theta$ is analytic in $\mathbb{C}$ and $\varphi(w) \neq 0$ is analytic in $\mathbb{C}^{*}$. Also, if we let

$$
Q(z)=z q^{\prime}(z) \varphi(q(z))=\gamma \frac{z q^{\prime}(z)}{q(z)},
$$

and

$$
g(z)=\theta(q(z))+Q(z)=1+\gamma \frac{z q^{\prime}(z)}{q(z)}
$$


from (3.12), we see that $Q(z)$ is a starlike function in $U$. From (3.12), we also have

$$
\Re\left\{\frac{z g^{\prime}(z)}{Q(z)}\right\}=\Re\left(1+\frac{z q^{\prime \prime}(z)}{q^{\prime}(z)}-\frac{z q^{\prime}(z)}{q(z)}\right)>0 \quad(z \in U)
$$

and then, by using Lemma 2.2 we deduce that the subordination (3.13) implies $h(z) \prec q(z)$, and the function $q$ is the best dominant of (3.13).

Taking $v=0, \eta=1, \gamma=1$ and $q(z)=\frac{1+A z}{1+B z}(-1 \leq B<A \leq 1)$ in Theorem 3.4, it is easy to check that the assumption (3.12) holds, hence we obtain the next result.

Corollary 3.5 Let $\mu \in \mathbb{C}^{*}$. Let $f \in A(p)$ and suppose that

$$
\frac{I_{p, \lambda}^{n} f(z)}{z^{p}} \neq 0 \quad(z \in U) .
$$

If

$$
1+\mu\left[\frac{z\left(I_{p, \lambda}^{n} f(z)\right)^{\prime}}{I_{p, \lambda}^{n} f(z)}-p\right] \prec 1+\frac{(A-B) z}{(1+A z)(1+B z)}
$$

then

$$
\left[\frac{I_{p, \lambda}^{n} f(z)}{z^{p}}\right]^{\mu} \prec \frac{1+A z}{1+B z},
$$

and $\frac{1+A z}{1+B z}$ is the best dominant of (3.15).

Putting $v=n=p=1, \eta=0, \gamma=\frac{1}{a b}\left(a, b \in \mathbb{C}^{*}\right), \mu=a$, and $q(z)=(1-z)^{-2 a b}$ in Theorem 3.4, then combining this with Lemma 2.6 we obtain the next result due to Obradovic et al. [8, Theorem 1].

Corollary 3.6 [8] Let $a, b \in \mathbb{C}^{*}$ such that $|2 a b-1| \leq 1$ or $|2 a b+1|$ leq 1 . Let $f \in A$ and suppose that $\frac{f(z)}{z} \neq 0$ for all $z \in U$. If

$$
1+\frac{1}{b}\left(\frac{z f^{\prime}(z)}{f(z)}-1\right) \prec \frac{1+z}{1-z}
$$

then

$$
\left(\frac{f(z)}{z}\right)^{a} \prec(1-z)^{-2 a b}
$$

and $(1-z)^{-2 a b}$ is the best dominant of (3.16).

Remark 3.7 For $a=1$, Corollary 3.6 reduces to the recent result of Srivastava and Lashin [19].

Putting $v=n=p=\gamma=1, \eta=0$ and $q(z)=(1+B z)^{\frac{\mu(A-B)}{B}}(-1 \leq A<B \leq 1)$ in Theorem 3.4, and using Lemma 2.6 we obtain the next result.

Corollary 3.8 Let $-1 \leq A<B \leq 1$ with $B \neq 0$, and suppose that $\left|\frac{\mu(A-B)}{B}-1\right| \leq 1$ or $\left|\frac{\mu(A-B)}{B}+1\right| \leq 1$. Let $f \in A$ such that $\frac{f(z)}{z} \neq 0$ for all $z \in U$, and let $\mu \in \mathbb{C}^{*}$. If

$$
1+\mu\left(\frac{z f^{\prime}(z)}{f(z)}-1\right) \prec \frac{1+[B+\mu(A-B)] z}{1+B z}
$$

then

$$
\left(\frac{f(z)}{z}\right)^{\mu} \prec(1+B z)^{\frac{\mu(A-B)}{B}}
$$

and $(1+B z)^{\frac{\mu(A-B)}{B}}$ is the best dominant of (3.17).

Putting $v=n=p=1, \eta=0, \gamma=\frac{\mathrm{e}^{i \tau}}{a b \cos \tau}\left(a, b \in \mathbb{C}^{*} ;|\tau|<\frac{\pi}{2}\right)$ and $q(z)=(1-z)^{-2 a b \cos \tau \mathrm{e}^{-i \tau}}$ in Theorem 3.4, we obtain the following result due to Aouf et al. [2, Theorem 1]. 
Corollary 3.9 [2] Let $a, b \in \mathbb{C}^{*}$ and $|\tau|<\frac{\pi}{2}$ and suppose that $\left|2 a b \cos \tau e^{-i \tau}-1\right| \leq 1$ or $\left|2 a b \cos \tau e^{-i \tau}+1\right| \leq$ 1. Let $f \in A$ and suppose that $\frac{f(z)}{z} \neq 0$ for all $z \in U$. If

$$
1+\frac{e^{i \tau}}{b \cos \tau}\left(\frac{z f^{\prime}(z)}{f(z)}-1\right) \prec \frac{1+z}{1-z}
$$

then

$$
\left(\frac{f(z)}{z}\right)^{a} \prec(1-z)^{-2 a b \cos \tau e^{-i \tau}}
$$

and $(1-z)^{-2 a b \cos \tau e^{-i \tau}}$ is the best dominant of (3.18).

Theorem 3.10 Let $q$ be univalent in $U$, with $q(0)=1$, let $\mu, \gamma \in C^{*}$ and let $\delta, \Omega, \nu, \eta \in C$ with $v+\eta \neq 0$. Let $f(z) \in A(p)$ and suppose that $f$ and $q$ satisfy the next two conditions:

$$
\frac{\nu I_{p, \lambda}^{n-1} f(z)+\eta I_{p, \lambda}^{n} f(z)}{(v+\eta) z^{p}} \neq 0 \quad(z \in U),
$$

and

$$
\Re\left(1+\frac{z q^{\prime \prime}(z)}{q^{\prime}(z)}\right)>\max \left\{0,-\Re\left(\frac{\delta}{\gamma}\right)\right\} \quad(z \in U) .
$$

If

$$
\psi(z)=\left[\frac{\nu I_{p, \lambda}^{n-1} f(z)+\eta I_{p, \lambda}^{n} f(z)}{(\nu+\eta) z^{p}}\right]^{\mu}\left[\delta+\gamma \mu\left(\frac{\nu z\left(I_{p, \lambda}^{n-1} f(z)\right)^{\prime}+\eta z\left(I_{p, \lambda}^{n} f(z)\right)^{\prime}}{\nu I_{p, \lambda}^{n-1} f(z)+\eta I_{p, \lambda}^{n} f(z)}-p\right)\right]+\Omega
$$

and

$$
\psi(z) \prec \delta q(z)+\gamma z q^{\prime}(z)+\Omega
$$

then

$$
\left[\frac{\nu I_{p, \lambda}^{n-1} f(z)+\eta I_{p, \lambda}^{n} f(z)}{(v+\eta) z^{p}}\right]^{\mu} \prec q(z),
$$

and $q$ is the best dominant of (3.22).

Proof Let us define the function $h$ by

$$
h(z)=\left[\frac{\nu I_{p, \lambda}^{n-1} f(z)+\eta I_{p, \lambda}^{n} f(z)}{(v+\eta) z^{p}}\right]^{\mu} \quad(z \in U) .
$$

According to (3.16), the function $h$ is analytic in $U$, and differentiating (3.20) logarithmically with respect to $z$, we obtain

$$
\frac{z h^{\prime}(z)}{h(z)}=\mu\left[\frac{\nu z\left(I_{p, \lambda}^{n-1} f(z)\right)^{\prime}+\eta z\left(I_{p, \lambda}^{n} f(z)\right)^{\prime}}{\nu I_{p, \lambda}^{n-1} f(z)+\eta I_{p, \lambda}^{n} f(z)}-p\right]
$$

and hence

$$
z h^{\prime}(z)=\mu\left[\frac{\nu I_{p, \lambda}^{n-1} f(z)+\eta I_{p, \lambda}^{n} f(z)}{(\nu+\eta) z^{p}}\right]^{\mu}\left[\frac{\nu z\left(I_{p, \lambda}^{n-1} f(z)\right)^{\prime}+\eta z\left(I_{p, \lambda}^{n} f(z)\right)^{\prime}}{\nu I_{p, \lambda}^{n-1} f(z)+\eta I_{p, \lambda}^{n} f(z)}-p\right] .
$$


Let us consider the next functions

$$
\begin{aligned}
& \theta(w)=\delta w+\Omega, \quad \varphi(w)=\gamma, \quad w \in \mathbb{C}, \\
& Q(z)=z q^{\prime}(z) \varphi(q(z))=\gamma z q^{\prime}(z) \quad(z \in U),
\end{aligned}
$$

and

$$
g(z)=\theta(q(z))+Q(z)=\delta q(z)+\gamma z q^{\prime}(z)+\Omega(z \in U) .
$$

From the assumption (3.20) we see that $Q$ is starlike in $U$ and that

$$
\Re\left(\frac{z g^{\prime}(z)}{Q(z)}\right)=\Re\left(\frac{\delta}{\gamma}+1+\frac{z q^{\prime \prime}(z)}{q^{\prime}(z)}\right)>0 \quad(z \in U),
$$

thus, by applying Lemma 2.2, the proof of Theorem 3.10 is completed.

Taking $q(z)=\frac{1+A z}{1+B z}(-1 \leq B<A \leq 1)$ in Theorem 3.10 and according to (3.5), the condition (3.20) becomes

$$
\max \left\{0 ;-\Re\left(\frac{\delta}{\gamma}\right)\right\} \leq \frac{1-|B|}{1+|B|} .
$$

Hence, for the special case $v=\gamma=1$ and $\eta=0$, we obtain the following result.

Corollary 3.11 Let $\delta \in \mathbb{C}$ with

$$
\max \{0 ;-\Re(\delta)\} \leq \frac{1-|B|}{1+|B|} .
$$

Let $f \in A(p)$, and suppose that $\frac{I_{p, \lambda}^{n-1} f(z)}{z^{p}} \neq 0$ for all $z \in U$, and let $\mu \in \mathbb{C}^{*}$. If

$$
\left[\frac{I_{p, \lambda}^{n-1} f(z)}{z^{p}}\right]^{\mu}\left[\delta+\mu\left(\frac{z\left(I_{p, \lambda}^{n-1} f(z)\right)^{\prime}}{I_{p, \lambda}^{n-1} f(z)}-p\right)\right]+\Omega \prec \delta \frac{1+A z}{1+B z}+\Omega+\frac{(A-B) z}{(1+B z)^{2}}
$$

then

$$
\left[\frac{I_{p, \lambda}^{n-1} f(z)}{z^{p}}\right]^{\mu} \prec \frac{1+A z}{1+B z}
$$

and $\frac{1+A z}{1+B z}$ is the best dominant of (3.24).

Taking $p=v=\gamma=n=1, \eta=0$ and $q(z)=\frac{1+z}{1-z}$ in Theorem 3.10, we obtain the following result.

Corollary 3.12 Let $f \in A$ such that $\frac{f(z)}{z} \neq 0$ for all $z \in U$, and let $\mu \in \mathbb{C}^{*}$. If

$$
\left(\frac{f(z)}{z}\right)^{\mu}\left[\delta+\mu\left(\frac{z f^{\prime}(z)}{f(z)}-1\right)\right]+\Omega \prec \delta \frac{1+z}{1-z}+\Omega+\frac{2 z}{(1-z)^{2}},
$$

then

$$
\left(\frac{f(z)}{z}\right)^{\mu} \prec \frac{1+z}{1-z}
$$

and $\frac{1+z}{1-z}$ is the best dominant of (3.25). 


\section{Superordination and sandwich results}

Theorem 4.1 Let $q$ be convex in $U$ with $q(0)=1$, let $\mu \in \mathbb{C}^{*}$ with $\Re(\mu)>0$. Let $f \in A(p)$ and suppose that $\frac{I_{p, \lambda}^{n} f(z)}{z^{p}} \in H[q(0) ; 1] \cap Q$. If the function

$$
\frac{\mu}{p}\left(\frac{I_{p, \lambda}^{n-1} f(z)}{z^{p}}\right)+\frac{p-\mu}{p}\left(\frac{I_{p, \lambda}^{n} f(z)}{z^{p}}\right)
$$

is univalent in the unit disc $U$, and

$$
q(z)+\frac{\lambda \mu}{p^{2}} z q^{\prime}(z) \prec \frac{\mu}{p}\left(\frac{I_{p, \lambda}^{n-1} f(z)}{z^{p}}\right)+\frac{p-\mu}{p}\left(\frac{I_{p, \lambda}^{n} f(z)}{z^{p}}\right),
$$

then

$$
q(z) \prec \frac{I_{p, \lambda}^{n} f(z)}{z^{p}}
$$

and $q$ is the best subordinant of (4.1).

Proof We define the function $g$ by

$$
g(z)=\frac{I_{p, \lambda}^{n} f(z)}{z^{p}} \quad(z \in U) .
$$

From the assumption of Theorem 4.1, the function $g$ is analytic in $U$. Differentiating (4.2) logarithmically with respect to $z$, we obtain

$$
\frac{z g^{\prime}(z)}{g(z)}=\frac{z\left(I_{p, \lambda}^{n} f(z)\right)^{\prime}}{I_{p, \lambda}^{n} f(z)}-p .
$$

After some computations, and using the identity (1.6) from (4.3), we get

$$
g(z)+\frac{\lambda \mu}{p^{2}} z g^{\prime}(z)=\frac{\mu}{p}\left(\frac{I_{p, \lambda}^{n-1} f(z)}{z^{p}}\right)+\frac{p-\mu}{p}\left(\frac{I_{p, \lambda}^{n} f(z)}{z^{p}}\right),
$$

and now, by using Lemma 2.5 we get the desired result.

Taking $q(z)=\frac{1+A z}{1+B z}(-1 \leq B<A \leq 1)$ in Theorem 4.1, we obtain the following corollary.

Corollary 4.2 Let $q$ be convex in $U$ with $q(0)=1$, let $\mu \in \mathbb{C}^{*}$ with $\Re(\mu)>0$. Let $f \in A(p)$ and suppose that $\frac{I_{p, \lambda}^{n} f(z)}{z^{p}} \in H[q(0), 1] \cap Q$. If the function

$$
\frac{\mu}{p}\left(\frac{I_{p, \lambda}^{n-1} f(z)}{z^{p}}\right)+\frac{p-\mu}{p}\left(\frac{I_{p, \lambda}^{n} f(z)}{z^{p}}\right)
$$

is univalent in $U$, and

$$
\frac{1+A z}{1+B z}+\frac{\lambda \mu}{p^{2}} \frac{(A-B) z}{(1+B z)^{2}} \prec \frac{\mu}{p}\left(\frac{I_{p, \lambda}^{n-1} f(z)}{z^{p}}\right)+\frac{p-\mu}{p}\left(\frac{I_{p, \lambda}^{n} f(z)}{z^{p}}\right),
$$

then

$$
\frac{1+A z}{1+B z} \prec \frac{I_{p, \lambda}^{n} f(z)}{z^{p}}
$$

and $\frac{1+A z}{1+B z}$ is the best subordinant of (4.4).

Using arguments similar to those of the proof of Theorem 3.10, and then by applying Lemma 2.4, we obtain the following result. 
Theorem 4.3 Let $q$ be convex in $U$ with $q(0)=1$, let $\mu, \gamma \in \mathbb{C}^{*}$, and let $\delta, \Omega, v, \eta \in \mathbb{C}$ with $v+\eta \neq 0$ and $\Re\left(\frac{\delta}{\gamma}\right)>0$. Let $f \in A(p)$ and suppose that $f$ satisfies the next conditions:

$$
\frac{\nu I_{p, \lambda}^{n-1} f(z)+\eta I_{p, \lambda}^{n} f(z)}{(v+\eta) z^{p}} \neq 0 \quad(z \in U),
$$

and

$$
\left[\frac{\nu I_{p, \lambda}^{n-1} f(z)+\eta I_{p, \lambda}^{n} f(z)}{(v+\eta) z^{p}}\right]^{\mu} \in H[q(0), 1] \cap Q .
$$

If the function $\psi$ given by (3.21) is univalent in $U$, and

$$
\delta q(z)+\gamma z q^{\prime}(z)+\Omega \prec \psi(z)
$$

then

$$
q(z) \prec\left[\frac{\nu I_{p, \lambda}^{n-1} f(z)+\eta I_{p, \lambda}^{n} f(z)}{(v+\eta) z^{p}}\right]^{\mu},
$$

and $q$ is the best subordinant of (4.5).

Combining Theorem 3.4 with Theorem 4.1 and Theorem 3.10 with Theorem 4.3, we obtain, respectively, the following two sandwich results.

Theorem 4.4 Let $q_{1}$ and $q_{2}$ be two convex functions in $U$ with $q_{1}(0)=q_{2}(0)=1$, let $\mu \in \mathbb{C}^{*}$ with $\Re(\mu)>0$. Let $f \in A(p)$ and suppose that $\frac{I_{p, \lambda}^{n} f(z)}{z^{p}} \in H[q(0), 1] \cap Q$. If the function

$$
\frac{\mu}{p}\left(\frac{I_{p, \lambda}^{n-1} f(z)}{z^{p}}\right)+\frac{p-\mu}{p}\left(\frac{I_{p, \lambda}^{n} f(z)}{z^{p}}\right)
$$

is univalent in the unit disc $U$, and

$$
q_{1}(z)+\frac{\lambda \mu}{p^{2}} z q_{1}^{\prime}(z) \prec \frac{\mu}{p}\left(\frac{I_{p, \lambda}^{n-1} f(z)}{z^{p}}\right)+\frac{p-\mu}{p}\left(\frac{I_{p, \lambda}^{n} f(z)}{z^{p}}\right) \prec q_{2}(z)+\frac{\lambda \mu}{p^{2}} z q_{2}^{\prime}(z)
$$

then

$$
q_{1}(z) \prec \frac{I_{p, \lambda}^{n} f(z)}{z^{p}} \prec q_{2}(z),
$$

and $q_{1}$ and $q_{2}$ are, respectively, the best subordinant and the best dominant of (4.6).

Theorem 4.5 Let $q_{1}$ and $q_{2}$ be two convex functions in $U$ with $q_{1}(0)=q_{2}(0)=1$, let $\mu, \gamma \in \mathbb{C}^{*}$, and let $\delta, \Omega, v, \eta \in \mathbb{C}$ with $v+\eta \neq 0$ and $\Re\left(\frac{\delta}{\gamma}\right)>0$. Let $f \in A(p)$ and suppose that $f$ satisfies the next conditions:

$$
\frac{\nu I_{p, \lambda}^{n-1} f(z)+\eta I_{p, \lambda}^{n} f(z)}{(v+\eta) z^{p}} \neq 0 \quad(z \in U),
$$

and

$$
\left[\frac{\nu I_{p, \lambda}^{n-1} f(z)+\eta I_{p, \lambda}^{n} f(z)}{(v+\eta) z^{p}}\right]^{\mu} \in H[q(0), 1] \cap Q .
$$

If the function $\psi$ given by (3.18) is univalent in $U$, and

$$
\delta q_{1}(z)+\gamma z q_{1}^{\prime}(z)+\Omega \prec \psi(z) \prec \delta z q_{2}(z)+\gamma z q_{2}^{\prime}+\Omega,
$$

then

$$
q_{1}(z) \prec\left[\frac{\nu I_{p, \lambda}^{n-1} f(z)+\eta I_{p, \lambda}^{n} f(z)}{(\nu+\eta) z^{p}}\right]^{\mu} \prec q_{2}(z),
$$

and $q_{1}$ and $q_{2}$ are, respectively, the best subordinant and the best dominant of (4.7). 
Remark 4.6 Putting $\lambda=1$ in the above results, we obtain the corresponding results for the $p$-valent Salagean integral operator $I_{p}^{n}$.

Acknowledgments The authors are grateful to the referees for their valuable suggestions.

Open Access This article is distributed under the terms of the Creative Commons Attribution License which permits any use, distribution, and reproduction in any medium, provided the original author(s) and the source are credited.

\section{References}

1. Ali, R.M.; Ravichandran, V.; Khan, M.H.; Subramanian, K.G.: Differential sandwich theorems for certain analytic functions. Far East J. Math. Sci. 15, 87-94 (2004)

2. Aouf, M.K.; Al-Oboudi, F.M.; Haidan, M.M.: On some results for $\lambda$-spirallike and $\lambda$-Robertson functions of complex order. Publ. Inst. Math. Belgrade 77(91), 93-98 (2005)

3. Bulboaca, T.: Classes of first order differential superordinations. Demonstr. Math. 35(2), 287-292 (2002)

4. Bulboaca, T.: A class of superordination-preserving integral operators. Indeg. Math. (N.S.) 13(3), 301-311 (2002)

5. Bulboaca, T.: Differential Subordinations and Superordinations. Recent Results. House of Scientific Book Publ., Cluj-Napoca (2005)

6. Miller, S.S.; Mocanu, P.T.: Differential Subordinations: Theory and Applications. Series on Monographs and Textbooks in Pure and Appl. Math. vol. 225. Marcel Dekker, New York (2000)

7. Miller, S.S.; Mocanu, P.T.: Subordinants of differential superordinations. Complex Variables 48(10), 815-826 (2003)

8. Obradovic, M.; Aouf, M.K.; Owa, S.: On some results for starlike functions of complex order. Publ. Inst. Math. (Beograd) (N.S.) 46(60), 79-85 (1989)

9. Obradovic, M.;Owa, S.: On certain properties for some classes of starlike functions. J. Math. Anal. Appl. 145, 357-364 (1990)

10. Patel, J.: Inclusion relations and convolution properties of certain subclasses of analytic functions defined by a generalized Salagean operator. Bull. Belg. Math. Soc. Simon Stevin 15, 33-47 (2008)

11. Royster, W.C.: On the univalence of a certain integral. Michigan Math. J. 12, 385-387 (1965)

12. Salagean, G.S.: Subclasses of univalent functions. Lecture Notes in Math. vol. 1013, pp. 362-372. Springer, Berlin (1983)

13. Shams, S.; Kulkarni, S.R.; Jahangiri, J.M.: Subordination properties for p-valent functions defined by integral operator. Int. J. Math. Math. Sci. 94572, 1-3 (2006)

14. Shanmugam, T.N.; Ravichandran, V.; Darus, M.; Sivasubramanian, S.: Differential sandwich theorems for some subclasses of analytic functions involving a linear operator. Acta Math. Univ. Comenianae 74(2), 287-294 (2007)

15. Shanmugam, T.N.; Ravichandran, V.; Sivasubramanian, S.: Differential sandwich theorems for some subclasses of analytic functions. J. Aust. Math. Anal. Appl. 3(1), 1-11 (2006)

16. Shanmugam, T.N.; Sivasubramanian, S.; Srivastava, H.M.: Differential sandwich theorems for certain subclasses of analytic functions involving multiplier transformations. Integral Transforms Spec. Funct. 17(12), 889-899 (2006)

17. Shanmugam, T.N.; Sivasubramanian, S.; Srivastava, H.M.: On sandwich theorems for some classes of analytic functions. Int. J. Math. Math. Sci. 29684, 1-13 (2006)

18. Singh, V.: On some criteria for univalence and starlikeness. Indian J. Pure Appl. Math. 34(4), 569-577 (2003)

19. Srivastava, H.M.; Lashin, A.Y.: Some applications of the Briot-Bouquet differential subordination. J. Inequal. Pure. Appl. Math. 6(2), 1-7 (2005)

20. Wang, Z.; Gao, C.; Liao, M.: On certain generalized class of non-Bazilevic functions. Acta Math. Acad. Proc. Nyircg. New Ser. 21(2), 147-154 (2005) 